\title{
Influência da educação integral na prevalência de transtorno do desenvolvimento da coordenaçáo em crianças em idade escolar
}

\author{
Thamires Francelino Mendonça de Melo ${ }^{a}$, Aline Midori Adati Kubotab, \\ Pedro Henrique Tavares Queiroz de Almeida ${ }^{\mathrm{b}}$, Tatiana Barcelos Pontes ${ }^{\mathrm{b}}$ \\ ${ }^{a}$ Secretaria de Estado de Saúde do Distrito Federal, Brasília, DF, Brasil \\ 'Universidade de Brasília - UnB, Brasília, DF, Brasil
}

\begin{abstract}
Resumo: Objetivos: Verificar a prevalência do Transtorno de Desenvolvimento da Coordenação (TDC) em crianças entre 7 e 10 anos de idade que participam do programa de educação integral e em crianças que cursaram apenas o ensino regular, avaliando a influência dos componentes de desempenho sobre o desenvolvimento motor relacionado ao TDC. Metodologia: A amostra foi constituída por 50 crianças, divididas em dois grupos conforme o tipo de educação recebida. Para avaliar o desenvolvimento neuropsicomotor foi utilizado o teste ABC do movimento, sendo a prevalência de TDC calculada conforme as normas britânicas para crianças de 4 a 16 anos de idade. A relação entre o tipo de educação e o desenvolvimento motor foi analisada pelo teste de Mann-Whitney e a relação entre componentes de desempenho foi analisada pelo Coeficiente de Correlação de Spearman. Resultados: A prevalência de TDC entre as crianças avaliadas foi de $8 \%$. Não foi observada relação significativa entre as variáveis tipo de educação e desempenho motor das crianças. Observou-se forte correlação entre o componente destreza manual e a presença de TDC e relação significativa entre a incidência de TDC e o gênero, sendo maior o número de meninas que apresentaram o transtorno. Conclusão: Resultados encontrados sugerem que o programa de educação integral pode não estar exercendo influência no desempenho das crianças, como preconizado. Esse fato pode estar relacionado à forma como o programa é estruturado nas escolas e com a organização do funcionamento e sugerem a necessidade de uma avaliação do programa de educação integral.
\end{abstract}

Palavras-chave: Ambiente Escolar, Desenvolvimento, Transtorno de Desenvolvimento da Coordenação.

\section{Influence of full-time education in the prevalence of developmental coordination disorder in school age children}

\begin{abstract}
Objectives: To investigate the prevalence of Developmental Coordination Disorder (DCD) among Brazilian children between 7 and 10 years of age participating in a full-time school program or attending regular school, by assessing the influence of different performance components on motor development related to DCD. Methods: The sample was composed of 50 children divided into two groups, according to the type of education received. Neuropsychomotor development was assessed using the Movement ABC-2 (MABC-2) Test, according to UK norms for 4-16 year olds. The prevalence of DCD was calculated according to the test results. The relationship between form of education and motor development was analyzed using the Mann-Whitney test; the correlation between performance components was analyzed by the Spearman Rank Order Correlation Coefficient (RHO). Results: DCD prevalence among the participating children was $8 \%$. The Mann-Whitney test showed no significant correlation between the variables type of education and motor performance of children. We observed a strong correlation between the hand dexterity component and the presence of DCD, with increased prevalence of the
\end{abstract}

Autor para correspondência:Tatiana Barcelos Pontes, Faculdade de Ceilândia, Universidade de Brasília, Centro Metropolitano, Conjunto A, Lote 01, CEP 72220-900, Brasília, DF, Brasil, e-mail: tatiana.pontes@ gmail.com

Recebido em 21/8/2013; Revisão em 1/4/2014; Aceito em 24/4/2014. 
disorder among girls. Conclusion: The results suggest that the full-time education programs conducted in Brazilian public schools are not providing proper stimuli to children's development, as advocated. This fact could be related with improper organization and lack of structuring in this educational modality in schools, indicating the necessity of proper evaluation of full-time programs.

Keywords: School Environment, Development, Developmental Coordination Disorder.

\section{Introdução}

A formação global do indivíduo se dá através do processo de desenvolvimento neuropsicomotor. Esse desenvolvimento refere-se ao surgimento de habilidades sensório-motoras, cognitivas, socioemocionais e de linguagem (ENGLE et al., 2007).

A aquisição de tais habilidades acontece numa sequência previsível, porém cada indivíduo possui um tempo próprio para o desenvolvimento, devido à influência de fatores ambientais, psicossociais e biológicos. Quando essa variação individual se mostra distante dos limites considerados normais, é caracterizada como desvio. Embora tais desvios sejam associados a desordens neurológicas, há casos em que as dificuldades motoras apresentam-se isoladamente, caracterizando o Transtorno do Desenvolvimento da Coordenação - TDC (SANTOS; DANTAS; OLIVEIRA, 2004).

O TDC é caracterizado por comprometimento motor que interfere nas atividades de vida diária e no desempenho acadêmico, não estando associado a transtornos neurológicos identificáveis (BARNHART et al., 2003). Crianças com TDC possuem habilidades de coordenaçáo motora que são substancialmente abaixo do esperado para idade (VAIVRE-DOURET, 2014), o que pode influenciar as atividades da vida diária, a participação em sala de aula e a interaçáo com os pares (DAVIS et al., 2007). Dificuldades em atividades como vestir-se, amarrar cadarços, escrita e habilidades com bola também podem estar presentes. Além disso, baixa autoestima, isolamento social e emocional e obesidade são potenciais consequências da doença a longo prazo (BURNS et al., 2009).

A etiologia do TDC ainda é indefinida. Porém algumas hipóteses são discutidas, como lesão neuronal, problemas pré-natais, perinatais e neonatais ou estímulos e oportunidades de desenvolvimento oferecidos pelo ambiente em que a criança está inserida (BARNHART et al., 2003; HILLIER, 2007).

Uma vez que crianças com TDC apresentam comprometimentos nos diferentes contextos em que estão inseridas, o ambiente escolar, por demandar diferentes habilidades motoras, deve auxiliar essas crianças a lidarem com esses problemas (VALENTINI et al., 2012). Dessa forma, o professor deve ser um observador ativo durante o desenvolvimento das atividades acadêmicas de seus alunos, identificando sinais indicativos de desordens motoras (SILVA et al., 2006).

Tendo em vista a importância das atividades educacionais para o desenvolvimento infantil e a existência de diferentes modalidades de educação disponíveis na rede pública de ensino, variando entre carga horária parcial e integral, o objetivo deste estudo foi verificar a prevalência de Transtorno do Desenvolvimento da Coordenaçáo entre dois grupos de crianças em idade escolar, observando também a relação entre diferentes tipos de educação oferecidos e o desempenho motor.

\section{Metodologia}

Foi realizado estudo de caráter exploratório, transversal, com crianças de idade entre 7 e 10 anos matriculadas em uma escola pública de uma regiáo administrativa do Distrito Federal.

A amostra utilizada foi não probabilística, do tipo intencional. Dois grupos foram definidos para efeito de comparação. Todas as crianças participantes do programa de educação integral foram convidadas a participar da pesquisa, cabendo aos pais e/ou responsáveis a autorização. A partir da quantidade de crianças que frequentavam a escola integral, crianças que cursavam apenas o ensino regular foram selecionadas, buscando-se pareá-las em relação ao sexo, idade e série.

Foram incluídas crianças que cursavam entre $\mathrm{o}$ $2^{\circ}$ e o $4^{\circ}$ ano do ensino fundamental em 2012 e que tinham frequentado a educação integral durante o ano de 2011 - para os participantes da educação integral.

Foram excluídas as crianças que apresentavam incapacidades previamente informadas que pudessem interferir em seu desenvolvimento neuropsicomotor.

Dados referentes ao desenvolvimento neuropsicomotor foram obtidos por meio do instrumento de avaliação $\mathrm{ABC}$ do Movimento, composto por uma bateria de testes motores e 
um checklist, aplicado a crianças de 3 a 16 anos de idade e dividido conforme a faixa etária. Os testes motores do $\mathrm{ABC}$ do movimento envolvem habilidades manuais, equilíbrio estático e dinâmico e habilidades com bola. O tempo de avaliaçáo do teste é de em média 30 minutos e a pontuação é atribuída de acordo com o rendimento da criança em cada atividade. Esse valor é convertido para a escala de pontuaçáo da bateria motora e assim o desempenho da criança é contabilizado.

A escala padrão do teste classifica o desenvolvimento em faixas de cores verde, amarelo ou vermelho. Essa classificação significa, respectivamente, desenvolvimento normal; em risco de apresentar problemas motores; ou com dificuldades motoras - caracterizando o Transtorno de Desenvolvimento da Coordenaçáo. A faixa verde corresponde a percentual acima de $15 \%$, a amarela, à pontuação entre 5\% e 15\%; e a vermelha, à pontuação inferior ou igual a 5\%. Para análise dos dados foram agrupadas as duas últimas faixas. Assim, foram consideradas como grupo de risco para o Transtorno de Desenvolvimento da Coordenação as crianças que apresentaram percentual menor que $15 \%$ no resultado da avaliação.

Para este estudo foi utilizado o teste completo, de acordo com a faixa etária avaliada. Cada criança foi avaliada uma única vez no decorrer do estudo. A avaliação das crianças ocorreu na própria escola, em espaço destinado à atividade.

\subsection{Procedimentos}

O projeto foi aprovado pelo Comitê de Ética em Pesquisa com Seres Humanos da Faculdade de Saúde da Universidade de Brasília, sob registro n. 182/11. A participação das crianças na pesquisa foi autorizada pelos pais, mediante assinatura do Termo de Concordância Livre e Esclarecido.

As crianças avaliadas foram separadas em dois grupos de comparação, sendo o Grupo 1 formado por crianças participantes do programa de educação integral e o Grupo 2 formado por crianças que frequentavam a escola em período regular.
Os participantes foram identificados por meio de nome, data de nascimento, idade no momento da avaliação, ano que estavam cursando e turma. Os dados foram organizados em planilhas do software Microsoft Excel 2010.

Para caracterização da população do estudo foram calculadas as medidas de estatística descritiva média, frequência e desvio padrão. Os testes de Mann-Whitney e o teste de correlação de Spearman foram utilizados para verificar a relaçáo entre o tipo de educação recebida e a correlação entre os componentes de desempenho motor e o escore final do teste, respectivamente.

\section{Resultados}

\subsection{Dados demográficos}

A amostra foi composta por 25 alunos que participaram do programa de educaçáo integral durante o ano de 2011 e 25 alunos que cursaram apenas o ensino regular no mesmo ano.

A idade média das crianças da amostra foi semelhante entre os grupos. O Grupo 1 apresentou idade média de 8,4 anos $(\mathrm{DP} \pm 1,04)$. A idade média do Grupo 2 foi de 8 anos (DP $\pm 0,88)$. A amostra foi considerada homogênea quanto ao gênero e distribuição dos participantes entre os anos do ensino fundamental (Tabela 1).

\subsection{Relação entre tipo da escola e a ocorrência de TDC}

Não foram encontradas relaçôes significativas entre o tipo da escola frequentada e o escore obtido pelas crianças de acordo com o teste de Mann-Whitney $(\mathrm{p}=0,807)$.

Em relação aos dados obtidos com o teste $\mathrm{ABC}$ do movimento, os dois grupos de crianças apresentaram a mesma distribuição entre os participantes quanto à classificação do teste, sendo observadas $84 \%$ das crianças com desenvolvimento normal, $8 \%$ em risco de apresentarem problemas no desenvolvimento

Tabela 1. Distribuição por gênero e período escolar entre os participantes.

\begin{tabular}{lccc}
\hline & Grupo 1 n (\%) & Grupo 2 n (\%) & $P$ \\
\hline Sexo feminino & $14(56 \%)$ & $16(64 \%)$ & $\mathbf{0 , 5 6 4}$ \\
Sexo masculino & $11(44 \%)$ & $09(36 \%)$ & \\
$\mathbf{3}^{\mathbf{0}}$ ano do ensino fundamental & $06(24 \%)$ & $09(36 \%)$ & \\
$\mathbf{4}^{\mathbf{0}}$ ano do ensino fundamental & $11(44 \%)$ & $10(40 \%)$ & $\mathbf{0 , 6 2 7 *}$ \\
$\mathbf{5}^{\mathbf{0}}$ ano do ensino fundamental & $08(32 \%)$ & $06(24 \%)$ & \\
\hline
\end{tabular}

*Teste de Chi-Quadrado de Pearson. 
e $8 \%$ com resultado indicativo de Transtorno de Desenvolvimento da Coordenação.

Ao se comparar a prevalência de casos de TDC entre os gêneros, foi observada diferença significativa $(\mathrm{p}=0,041)$ entre os indivíduos do sexo masculino e feminino, com maioria dos casos de risco e com indicativo de TDC entre meninas. Dentre as quatro crianças que apresentaram indicativo de TDC, três eram do sexo feminino.

Os dados relativos ao risco de desenvolvimento de TDC entre a população participante estão na Tabela 2.

\subsection{Impacto dos componentes sobre o escore total do $\mathrm{mABC}$}

Observou-se ainda a influência do escore obtido na avaliação de cada componente de desempenho sobre o resultado final da avaliaçáo por meio do coeficiente de correlaçáo de postos de Spearman.

As atividades de destreza manual demonstraram maior influência no resultado final do teste (coeficiente de determinação de $74,6 \%$ ), seguidas pelas atividades relacionadas às habilidades com bola e pelos testes de equilíbrio (coeficientes de determinação de 44,35\% e $28,4 \%$, respectivamente), indicando que melhores níveis de desempenho nessas habilidades estão relacionados a altos escores do $\mathrm{mABC}(\mathrm{p}=0,001)$ (Tabela 3).

\section{Discussão}

Estima-se que de $5 \%$ a $8 \%$ das crianças em idade escolar do mundo inteiro possuem TDC (BARNHART et al., 2003). Embora estudos nacionais relatem prevalência maior entre crianças na mesma faixa etária dos participantes deste estudo, variando entre $10,6 \%$ e $11,8 \%$ da população estudada (PELLEGRINI et al., 2006; SOUZA et al., 2007), o presente estudo identificou a prevalência de TDC em $8 \%$ das crianças participantes, corroborando estimativas internacionais.

Observou-se relaçẫo significativa entre a incidência de TDC e o gênero, sendo maior o número de meninas que apresentaram o transtorno. Tal resultado é similar ao relatado por Silva e Beltrame (2013), porém mostra-se diferente dos resultados de pesquisas internacionais, onde a prevalência de TDC é majoritária entre crianças do sexo masculino (SMITSENGELSMAN; NIEMEIJER; VAN GALEN, 2001; BARNHART et al., 2003; KIRBY; SUDGEN, 2007; HILLIER, 2007).

Dentre as crianças identificadas com Transtorno do Desenvolvimento da Coordenação, todas apresentaram maior dificuldade nas atividades de destreza manual. Essas foram representadas no teste por atividades que exigiam habilidades gráficas e habilidades funcionais, como exemplo as atividades de alinhavo e encaixe de pinos. É estimado que as dificuldades em atividades que exigem habilidades motoras finas, como a escrita, estão presentes em $10 \%$ a $30 \%$ das crianças em idade escolar (FEDER; MAJNEMER, 2007; CHANG; YU, 2010).

Pela presença majoritária de atividades que exigem habilidades motoras finas na escola, ela se torna local propício para observaçáo das crianças e identificação dessas dificuldades. A escola é dos ambientes em que a criança fica na maior parte de seu tempo e como tal ela deve fornecer estímulos necessários ao desenvolvimento das capacidades e formação integral da criança (GOODGOLD, 1983; SUMMERS, 2001).

Tabela 2. Desempenho, risco e indicativo de TDC entre crianças avaliadas - divisão por grupos e gênero.

\begin{tabular}{|c|c|c|c|c|}
\hline & $\begin{array}{c}\text { Grupo 1* } \\
\mathbf{n}\end{array}$ & $\begin{array}{c}\text { Grupo } 2 * \\
\text { n }\end{array}$ & $\begin{array}{c}\text { Sexo masculino** } \\
\mathrm{n}\end{array}$ & $\begin{array}{c}\text { Sexo feminino** } \\
\mathbf{n}\end{array}$ \\
\hline Desempenho normal $(\mathrm{mABC}>16)$ & $21(84 \%)$ & $21(84 \%)$ & $18(90 \%)$ & $24(80 \%)$ \\
\hline Risco/ suspeita de TDC (mABC 6 a 15) & $02(8 \%)$ & $02(8 \%)$ & $01(5 \%)$ & $03(10 \%)$ \\
\hline Indicativo de TDC $(\mathrm{mABC}<5)$ & $02(8 \%)$ & $02(8 \%)$ & $01(5 \%)$ & $03(10 \%)$ \\
\hline
\end{tabular}

$* \mathrm{p}=0,807 ; * \mathrm{p}=0,041$.

Tabela 3. Coeficiente de correlação de Spearman entre o escore total do mABC e o escore obtido em cada componente da avaliação.

\begin{tabular}{|c|c|c|c|c|}
\hline & & 2 & 3 & 4 \\
\hline 1. $\mathrm{mABC}$ total & - & $0,844^{*}$ & $0,666^{*}$ & $0,533 *$ \\
\hline 2. mABC destreza manual & & - & $0,340 * *$ & $0,208 * * *$ \\
\hline 3. mABC habilidade com bola & & & - & $0,315 * *$ \\
\hline 4. mABC equilíbrio & & & & - \\
\hline
\end{tabular}

$* \mathrm{p}<0,001 ; * * \mathrm{p}<0,05 ; * * * \mathrm{p}>0,05$. 
Além da prevalência de TDC, observou-se a relação entre o tempo de escola e as atividades desenvolvidas com o desempenho motor das crianças. Para isso, utilizou-se como medida de comparação a participação, ou não, no programa de educação integral.

Em ambos os grupos, $8 \%$ das crianças foram identificadas com desempenho abaixo do esperado, apresentando risco de problemas em seu desenvolvimento motor. O mesmo resultado foi observado em relaçáo à presença de Transtorno de Desenvolvimento da Coordenação, que apresentou prevalência de $8 \%$ nos grupos. O programa de educação integral tem como objetivo ampliar e estimular as possibilidades de desenvolvimento em todos os âmbitos. Entretanto, o estudo demonstrou não haver diferenças significativas entre o desempenho de crianças participantes e não participantes do programa de educação integral.

Tal resultado sugere que o programa de educaçáo integral pode náo estar exercendo influência no desempenho das crianças, como preconiza seu objetivo. Esse fato pode estar relacionado com a forma como o programa é estruturado nas escolas e com a organização do funcionamento.

Dentre as hipóteses levantadas para a ocorrência de tal resultado é importante ressaltar que as crianças avaliadas no presente estudo são acompanhadas por bolsistas do governo do Distrito Federal (GDF), os quais muitas vezes não possuem a preparação necessária para trabalhar com crianças e não estão cursando a graduação em área relacionada à educação, muitas vezes não possuindo conhecimentos sobre desenvolvimento infantil. Por vezes, o programa de educação integral não dispóe de um espaço adequado para ser oferecido e a escola em que ele está inserido não possui recursos suficientes para que as atividades sejam desenvolvidas da melhor maneira. Existem alguns problemas conhecidos, como falta de salas, demanda grande de alunos para o número de profissionais, falta de preparação para desenvolver atividades que estimulem o desenvolvimento das crianças de acordo com suas particularidades e necessidades (GONÇALVES, 2006).

Outra possível explicação é que os grupos estudados são de apenas uma escola, de uma única região administrativa do Distrito Federal, portanto podem apresentar características socioculturais semelhantes, o que pode ter interferido na comparação entre os grupos (VALENTINI et al., 2012). O tamanho amostral (50 crianças) pode também ser um fator limitante do estudo, entretanto, cabe ressaltar que foram incluídas na pesquisa todas as crianças que estavam na educação integral e cuja participação foi aprovada.

Não foram encontrados outros estudos que verificassem a associação do desenvolvimento infantil com o programa de educaçáo integral. Algumas hipóteses para esse fato podem estar relacionadas a ele ser um programa relativamente novo, ou um tema de interesse maior dos profissionais da educação, cujo escopo de investigação centra-se nos aspectos metodológicos (LIMA, 2011; PEREIRA, 2011; VILLEROY, 2011).

\section{Conclusão}

A avaliação do desenvolvimento infantil é importante para a identificação de déficits que comprometam a participação e engajamento da criança em atividades significativas e necessárias para seu desenvolvimento neuropsicomotor.

A identificação de tais dificuldades faz-se importante não só para a indicação de tratamento e estimulação adequados, mas também para a adoção de medidas preventivas que permitam a identificaçáo precoce de impactos negativos sobre o desenvolvimento motor e favoreçam a elaboração de estratégias que minimizem sua ocorrência entre essa população. Uma vez que a maior parte das atividades escolares consiste de tarefas motoras finas, como colorir, recortar e escrever, atrasos no desenvolvimento motor contribuem negativamente para o desempenho escolar de crianças com TDC.

Além da importância da detecção de atrasos no desenvolvimento motor, observa-se que embora a modalidade de educação integral possa apresentar maior oportunidade de estímulos ao desenvolvimento infantil, não foram observadas diferenças significativas entre o tipo de educação e o risco de déficits no desenvolvimento motor, fato que sugere a necessidade de maiores investigaçóes sobre a qualidade dos estímulos fornecidos para a criança durante o período escolar.

É necessário ressaltar que são raros os estudos sobre TDC no Brasil, especialmente no que se refere a uma população assintomática, o que reforça a importância do estudo realizado e evidencia a necessidade de estudos de base populacional sobre o tema em diferentes regiōes do país e em diferentes contextos sociais.

\section{Referências}

BARNHART, R. C. et al. Developmental coordination disorder. Physical Therapy: Journal of the American Physical Therapy Association, Vancouver, v. 83, n. 8, p. 722-731, 2003. 
BURNS, Y. R. et al. Motor coordination difficulties and physical fitness of extremely-low-birthweight children. Developmental Medicine \& Child Neurology, Malden, v. 51, n. 2, p. 136-42, 2009. PMid:18811704. http:// dx.doi.org/10.1111/j.1469-8749.2008.03118.x

CHANG, S. H.; YU, N. Y. Characterization of motor control in handwriting difficulties in children with or without developmental coordination disorder. Developmental Medicine \& Child Neurology, Malden, v. 52, n. 3, p. 244-250, 2010. PMid:20002122. http:// dx.doi.org/10.1111/j.1469-8749.2009.03478.x

DAVIS, N. M. et al. Developmental coordination disorder at 8 years of age in a regional cohort of extremely-low-birthweight or very preterm infants. Developmental Medicine \& Child Neurology, Malden, v. 49, n. 5, p. 325-30, 2007. PMid:17489804. http:// dx.doi.org/10.1111/j.1469-8749.2007.00325.x

ENGLE, P. L. et al. Strategies to avoid the loss of developmental potential in more than 200 million children in the developing world. Lancet, London, n. 369, p. 229-242, 2007.

FEDER, K. P.; MAJNEMER, A. Handwriting development, competency, and intervention. Developmental Medicine \& Child Neurology, Malden, v. 49, n. 4, p. 312-317, 2007. PMid:17376144. http:// dx.doi.org/10.1111/j.1469-8749.2007.00312.x

GOODGOLD, S. A. Handwriting movement quality in prekindergarten and kindergarten children. Archives of Physical Medicine and Rehabilitation, Reston, v. 64, n. 10, p. 471-5, Oct. 1983.

GONÇALVES, A. S. Reflexôes sobre educação integral e escola de tempo integral. Cadernos CENPEC, São Paulo, v. 1, n. 2, p. 129-135, 2006.

KIRBY, A.; SUDGEN, D. A. Children with developmental coordination disorders. Journal of the Royal Society of Medicine, London, v. 100, n. 4, p. 182-186, 2007. PMid:17404341 PMCid:PMC1847727. http://dx.doi. org/10.1258/jrsm.100.4.182

LIMA, B. P. M. O Projeto Educação Integral e Inclusão social no contexto da educação integral e escola de tempo integral: estudo de caso. 2011. 89 f. Monografia (Licenciatura em Pedagogia)-Universidade de Brasília, Brasília, 2011.

HILLIER, S. Intervention for children with developmental coordination disorder: a systematic review. The Internet Journal of Allied Health Sciences and Practice, Orlando, v. 5, n. 3, p. 1-11, 2007.

PELLEGRINI, A. M. et al. Dificuldades motoras em crianças de 9-10 anos de idade: seriam os meninos mais descoordenados? In: Pinho SZ, Saglietti JR, editors.
Núcleos de Ensino da UNES. São Paulo: Cultura Acadêmica, 2006. p. 77-88. Disponível em: <http:// www.unesp.br/prograd/PDFNE2006/artigos/capítulo1/ dificuldades.pdf $>$. Acesso em: 13 dez. 2012.

PEREIRA, M. V. S. Um estudo exploratório sobre a educação integral na Escola Classe 614 de Samambaia-DF. 2011. 82 f. Monografia (Licenciatura em Pedagogia)-Universidade de Brasília, Brasília, 2011.

SANTOS, S.; DANTAS, L.; OLIVEIRA, J. A. Desenvolvimento motor de crianças, idosos e de pessoas com transtorno da coordenaçáo. Revista Paulista de Educação Física, São Paulo, v. 18, p. 33-44, 2004.

SILVA, J. A. O. et al. Teste mabc: aplicabilidade da lista de checagem na região sudeste do Brasil. Revista Portuguesa de Ciências do Desporto, Porto, v. 6, n. 3, p. 356-361, 2006.

SILVA, J.; BELTRAME, T. S. Indicativo de Transtorno do Desenvolvimento da Coordenação de escolares com idade entre 7 e 10 anos. Revista Brasileira de Ciências do Esporte, Florianópolis, v. 35, n. 1, p. 3-14, 2013.

SMITS-ENGELSMAN, B. C. M.; NIEMEIJER, A. S.; VAN GALEN, G. P. Fine motor deficiencies in children diagnosed as DCD based on poor grapho-motor ability. Human Movement Science, Amsterdam, v. 20, n. 1-2, p. 161-182, 2001. http://dx.doi.org/10.1016/ S0167-9457(01)00033-1

SOUZA, C. et al. O teste ABC do movimento em crianças de ambientes diferentes. Revista Portuguesa de Ciências do Desporto, Porto, v. 7, n. 1, p. 36-47, 2007.

SUMMERS, J. Joint laxity in the index finger and thumb and its relationship to pencil grasps used by children. Australian Occupational Therapy Journal, Malden, v. 48, n. 3, p. 132-141, 2001. http://dx.doi. org/10.1046/j.0045-0766.2001.00247.x

VAIVRE-DOURET, L. Developmental coordination disorders: state of art. Neurophysiologie Clinique, Montpellier, v. 44, n. 1, p. 13-23, 2014. PMid:24502901. http://dx.doi.org/10.1016/j.neucli.2013.10.133

VALENTINI, N. C. et al. Prevalência de déficits motores e desordem coordenativa desenvolvimental em crianças da regiấo Sul do Brasil. Revista Paulista de Pediatria, São Paulo, v. 30, p. 377-384, 2012. http://dx.doi.org/10.1590/ S0103-05822012000300011

VILLEROY, G. C. Um relato de vivência com a educação integral numa escola pública do Distrito Federal: CAIC Juscelino Kubitschek de Oliveira - Núcleo Bandeirante - DF. 2011. 64 f. Monografia (Licenciatura em Pedagogia)-Universidade de Brasília, Brasília, 2011. PMid:21721636.

\section{Contribuição dos Autores}

Thamires Francelino Mendonça de Melo: concepçáo e redação do texto, coleta de dados; Aline Midori Adati Kubota: coleta de dados, redação do texto; Pedro Henrique Tavares Queiroz de Almeida: análise estatística, redação e revisão do texto; Tatiana Barcelos Pontes: concepção da metodologia, análise dos resultados, redação e revisão do texto. 